\title{
Formules scientifiques pour l'optimisation de la réponse acoustique du tambour d'appel africain
}

\author{
J.H. Talla Mbé ${ }^{1,2}$, P. Woafo ${ }^{2}$, and J. Fame N dongo ${ }^{3}$ \\ ${ }^{1}$ Unitéde recherche de Matière Condensée d'Eletronique \& de Traitement du Signal, Faailté de Saiences, \\ Département de Physique, Univesité de Dschang Cameraun \\ ${ }^{2}$ Laborataire de Modalisation \& de Simlation en Ingérieie, Biomimátisme \& Protaypes, Faaulté de Säres, \\ Département de Physique, Université de Yaandé 1, Cameroun \\ ${ }^{3}$ Département deCommmications africaines, EcoleSupéieure des Säenes \& Tedriques del’Information \& de la \\ Comminication, Univesité de Yaandé II, Cameraun.
}

${ }^{*}$ Corresponding author, E-mail: pwoafo1@yahoofr

\begin{abstract}
Résumé
Le tambour d'appel africain (TAA) est le véhicule sonore de l'existence africaine. Il est associé à de nombreuses expressions traditionnelles et cultuelles. Le processus de fabrication présente quelques inconvénients : durée longue, difficulté à fabriquer des tambours acoustiquement identiques, pauvreté tonale et portée acoustique limitée. Dans le but d'apporter quelques solutions aux inconvénients susmentionnés, nous établissons les équations dynamiques du fonctionnement du TAA grâce aux lois de la mécanique et de l'acoustique. Les fréquences propres de vibration des lèvres et de la cavité sont obtenues. D eux formules scientifiques qui permettent d'optimiser la réponse acoustique des TAAs sont établies. Elles peuvent servir comme normes de fabrication afin d'obtenir des puissances sonores maximales et de gérer convenablement les intervalles musicaux. Les résultats théoriques sont validés par des mesures expérimentales et des tests dans des ateliers de fabrication.
\end{abstract}

Mots clés. Tambour d'appel africain, fréquences propres, formules scientifiques, optimisation de la fabrication.

\begin{abstract}
The African calling drum is a sound instrument associated to various traditional and cultural expressions in Africa. Its fabrication process presents some limits: long duration, difficulty in fabricating acoustically identical drums, tonal poverty and limited sound range. In order to find some solutions to these limits, dynamical equations describing the functioning of the African calling drum are derived using mechanical and acoustical laws. The natural frequencies of vibration of the drum shells and drum cavity are obtained. They are used to obtain formula for the fabrication of drums with maximal sound powers and convenient management of the musical intervals. The theoretical results are validated by the experiment and tests in some fabrication sites.
\end{abstract}

Keywords. African calling drum, natural frequencies, scientific formula, optimization of the fabrication.

Received:_10_/_04_/2019_

Accepted:_28_/_04_/2019

DOI: https:/ / dx.doi.org/ 10.4314/ jcas.v15i1.5

(C) TheAuthors This work is licensed under theCreativeCammons Attribution 4.0 Intemational Licene 


\section{Introduction}

Téléphone tambour ou tambour télégraphe, le tambour d'appel africain est un moyen de communication d'origine négro-africaine révélée en 1876 aux colons européens confondus par l'hermétisme de ce système de communication spécial développé et utilisé par des cultures précoloniales en zones forestières. Quand des expéditions européennes arrivèrent dans ces zones pour explorer la forêt primaire; elles étaient surprises de la connaissance de leur venue et de leurs inventions. Sur diverses contrées et sur de longues distances, le tambour télégraphe communique les bonnes et mauvaises nouvelles. D u simple moyen de communiquer des messages d'un village à l'autre, aux solennités marquant les étapes importantes d'une vie, le tambour résonne en s'enrobant d'un caractère cérémonial, officiel, métaphysique, symbolique et esthétique. S’il sert à la communication avant le divertissement, il est à noter qu'il participe de la didactique en éduquant et en enseignant le clan à travers un langage qui se module selon la forme de l'instrument et la frappe du tambourinaire qui joue sa percussion dans des conditions bien précises. Sans support d'écriture, le tambour raconte le passé des tribus, de génération en génération (Eno Belinga (1966), FAO (2002)).

D epuis des millénaires, le tambour d'appel africain règle la vie artistique et mystique en Afrique, et constitue le véhicule de l'existence africaine. Bien qu'aujourd'hui sacrifié sur l'autel des nouveaux instruments et autres moyens de télécommunications modernes, il ne manque pas d'intérêt pour ceux qui aiment la percussion traditionnelle et ce moyen de communication qui reste d'actualité. En effet, il a une portée acoustique qui peut aller au-delà de $10 \mathrm{~km}$ et peut transmettre des messages codés et compris seulement par une certaine catégorie de personnes initiées.
La Figure 1 présente un exemple de tambour d'appel africain (TAA). Il est fabriqué à partir d'un tronc d'arbre évidé horizontalement et pas verticalement, traversé par une fente longitudinale qui laisse tombante deux lèvres d'épaisseurs asymétriques sur lesquelles l'émetteur ou le tambourinaire, à l'aide de deux baguettes, frappe pour diffuser des messages codés ou des mélodies populaires. La lèvre mâle (la moins épaisse) émet un son grave alors que la femelle (la plus épaisse) émet un son aigu. Chacune se termine au niveau de la fente par une languette médiane.

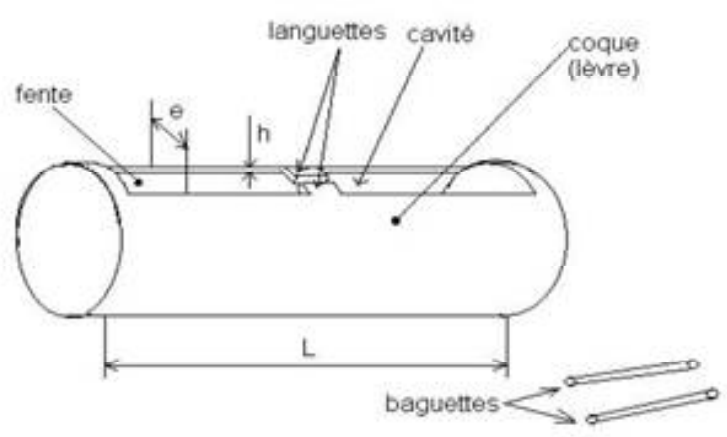

Figure 1: Le tambour d'appel africain (TAA) et ses différentes parties. e : ouverture des fentes et $\mathrm{h}$ : épaisseur de la coque.

Le TAA fait partie intégrante de la culture des peuples Bantu située en majorité dans la partie centrale de l'Afrique (Alexandre (1967), Ella (2015), Bingono-Bingono (2018)). On les trouve en l'occurrence dans les pays comme le Cameroun, l'Angola, le Burundi, les Iles Comores, le Congo, le Gabon, la Guinée-équatoriale, la République Centrafricaine, la République D émocratique du Congo, le Rwanda et la Zambie. Ces peuples ont des langues locales tonales constituées de deux tons dominants (le haut et le bas ou encore le ton aigu ou femelle et le ton grave ou mâle) auquel viennent s'y ajouter les tons montant, descendant, flottant, etc. On peut citer à titre illustratif la langue Béti au Cameroun qui a six tons, l'Umbundu d'Angola qui a trois, le Shiwa du G abon qui a quatre, etc. Les deux tons dominants de ces langues peuvent être rapprochés aux tons 
du TAA. En effet, le TAA produit une gamme de sons assez étendue dominée par deux sons principaux distants d'une tierce réglée lors de sa fabrication en faisant une différence d'épaisseur entre les deux lèvres. Les autres tons dépendent surtout de la manière dont l'instrument est percuté. Le TAA, par ces deux lèvres, peut être utilisé pour articuler les langues bantu. C'est pourquoi on l'appelle souvent tambour parlant.

Le TAA parle. Disjointe de la parole orale, la parole tambourinée est produite par lemouvement des baguettes et de la bille de bois évidée, activée par le joueur. Le TAA rythme et martèle les mots comme pour les inscrire dans les consciences de ceux qui parviennent à les décrypter. Ces mots expriment l'état d'âme du joueur face à la situation (joie ou malheur) que traverse le peuple. Le langage tambouriné est finalement une profusion des techniques rythmées produisant des sons et un langage parlé. Il a donc deux fonctions principales. La première est d'ordre communicationnel : c'est un instrument de signalisation, d'information, d'annonce d'un évènement. C'est une sorte de télécommunication sans courant électrique, ni réseau satellitaire. La deuxième fonction est d'ordre ludique : c'est un instrument de musique, modèle d'expression et de communication artistique. Il faut par ailleurs noter qu'à ces deux fonctions principales, on peut associer d'autres fonctions connexes : symbolique, spirituelle, métaphysique, politique, littéraire, esthétique, etc. C'est ainsi qu'au Soudan, le TAA fait partie des insignes royaux. De même, au Cameroun, il est utilisé pour faire des discours, pour annoncer des évènements (mariage, naissance, décès, grand rassemblement, alerte), pour faire des reportages sportifs comme la lutte traditionnelle afin de permettre à ceux qui n'ont pas pu se rendre sur le site du spectacle de le vivre. Il remplace les applaudissements lorsqu'une parole de sagesse est prononcée ou pour marquer la fin d'un discours, etc.
Toutes ces fonctions se manifestent à travers la réponse acoustique du TAA activée avec dextérité, habilité et rapidité par le joueur.

Pour fabriquer le TAA, les artisans africains disposent d'un matériel adéquat qui leur permet d'évider la bille de bois, de dimensionner les lèvres et les languettes. L'état final est jugé par le son que l'on perçoit suivi des réajustements jusqu'à l'obtention d'un son recherché. Le processus de fabrication se fait donc par tâtonnement et présente plusieurs inconvénients : - durée du processus, - difficulté à obtenir des sons de fréquences initialement choisies, - difficulté à fabriquer deux TAAs acoustiquement identiques, - difficulté à produire des sons de même fréquence en utilisant des billes de bois de dimensions différentes ou issues des espèces différentes de bois, - pauvreté tonale et portée acoustique limitée quand ils ne sont pas de grande taille. Ce dernier inconvénient rend difficile la miniaturisation des TAAs en vue de contribuer à la sauvegarde de l'environnement (en effet, pour fabriquer des tambours avec des portées assez grandes et produire des sons de fréquences particulières, l'artisan africain fait usage des billes de bois issues des arbres ayant mis de nombreuses années pour atteindre la taille et le diamètre souhaités).

LeTAA est donc un système acoustique complexe qui interagit avec l'homme et pour lequel la description par les lois de la mécanique reste un défi scientifique qui mérite d'être relevé.

D ans le but d'apporter quelques solutions aux inconvénients mentionnés ci-haut, une équipe pluridisciplinaire constituée des chercheurs camerounais s'est engagée dans le projet de modernisation, d'optimisation et de miniaturisation du TAA (Fame Ndongo (2010)). L'objectif du présent article vise à répondre aux questions suivantes. A partir des lois de la mécanique et de l'acoustique, comment établir les équations dynamiques du fonctionnement du TAA ? Arrive-t-on à obtenir des fréquences propres de 
vibration des lèvres et de la cavité ? Q uelles formules scientifiques s'établissent-t-elles pour optimiser la réponse acoustique du TAA ? Comment les mesures expérimentales et les tests dans les ateliers de fabrication arrivent-t-elles à valider les résultats théoriques de la recherche ? La section 2 propose une modélisation mécanique du TAA, en ressort ses équations dynamiques, et donne des informations sur les méthodes mathématiques et expérimentales utilisées. Dans la section 3, les fréquences de vibration des différents sous-systèmes du TAA sont établies et permettent de déduire les formules scientifiques pour la fabrication $d u$ TAA. La validation expérimentale de ces formules est également effectuée. La conclusion est donnée en section 4.
Chaque lèvre est considérée comme une coque cylindrique (Figure 2). En utilisant les hypothèses de coques minces de $D$ onnell et en négligeant les effets de la languette, le système d'équations décrivant la dynamique de chaque lèvre est donné par (D onnell (1934), Von Karman and Tsien (1941), Talla Mbé (2007)) :

$$
\begin{aligned}
& \left\{\begin{array}{l}
D \Delta \Delta W+\frac{1}{R} \frac{\partial^{2} F}{\partial z^{2}}+m \ddot{W}=L(F, W)+f_{\text {ext }} \\
\Delta \Delta F-\frac{h E}{R} \frac{\partial^{2} W}{\partial z^{2}}=-\frac{h E}{2} L(W, W)
\end{array}\right. \\
& \text { avec: } \Delta(\bullet)=\frac{1}{R^{2}} \frac{\partial^{2}(\bullet)}{\partial \theta^{2}}+\frac{\partial^{2}(\bullet)}{\partial z^{2}} \\
& L(W, F)=\frac{1}{R^{2}} \frac{\partial^{2} F}{\partial \theta^{2}} \frac{\partial^{2} W}{\partial z^{2}}+\frac{1}{R^{2}} \frac{\partial^{2} F}{\partial z^{2}} \frac{\partial^{2} W}{\partial \theta^{2}}-2 \frac{1}{R^{2}} \frac{\partial^{2} F}{\partial z \partial \theta} \frac{\partial^{2} W}{\partial \theta \partial z}
\end{aligned}
$$
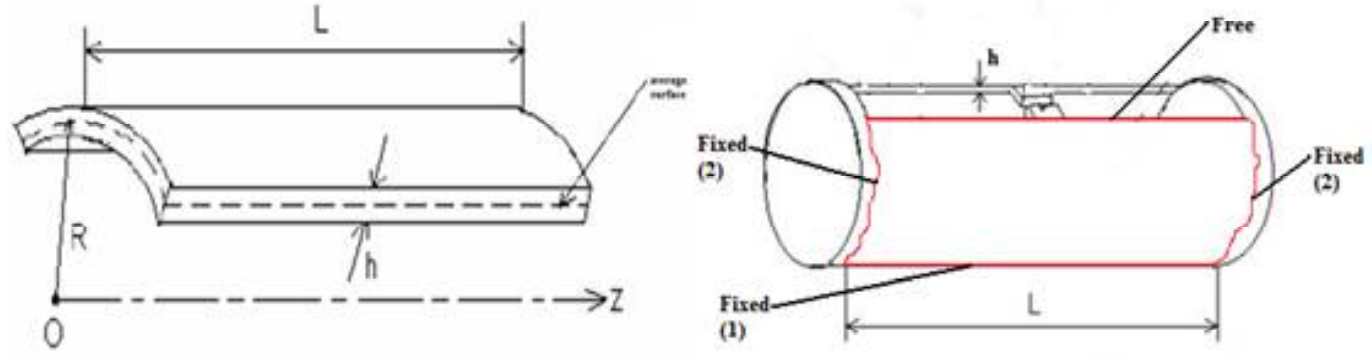

Figure 2 : (Gauche) : Une lèvre ou coque cylindrique. (D roite) : Les conditions aux limites. $\mathrm{R}$ : rayon de la coque, $\mathrm{h}$ : épaisseur de la coque ou de la lèvre, $\mathrm{L}:$ longueur de la coque.

\section{Méthodologie : Modélisation, méthodes mathématiques et expénimentales.}

\subsection{Modélisation}

Du point de vue du physicien, le TAA est formé de deux sous-systèmes : les coques et la cavité. La dynamique des coques a faitl'objet de plusieurs études dans le domaine de l'ingénierie et de la musique (D onnell (1934), Von Karman and Tsien (1941), Sanders (1963), Bezukhov (1987), Thomas (2005)). Mais s'agissant de celles des instruments de musique à surface de percussion cylindrique, elle reste à explorer. D 'où l'originalité de cette partie du travail qui ambitionne d'utiliser les lois de la mécanique pour modéliser le comportement vibratoire des coques du TAA et de sa cavité.
Les deux points renvoient à la dérivée seconde par rapport au temps. $F$ est la fonction d'Airy, $W$ est le déplacement transversal de la lèvre et $R$ son rayon. $\theta$ et $z$ sont respectivement l'angle et la cote en coordonnées cylindriques. $v$ et $E$ représentent respectivement le coefficient de Poisson et le module d'Young du matériau constituant la lèvre. Le paramètre $f_{\text {ent }}$ représente l'excitation extérieure produite par les baguettes.

Il faut ajouter les conditions aux limites à ces équations de la dynamique des lèvres. A cet effet, nous supposons que chaque lèvre a la configuration suivante : trois extrémités fixées et une extrémité libre, comme le montre la Figure 2. Mathématiquement, cela se traduit par : 
- Extrémité fixée 1: $\left\{\begin{array}{c}W=0 \\ \frac{1}{R^{2}} \frac{\partial^{2} W}{\partial \theta^{2}}+v \frac{\partial^{2} W}{\partial z^{2}}=0\end{array}\right.$

- Extrémité fixée 2: $\left\{\begin{array}{c}W=0 \\ v \frac{1}{R^{2}} \frac{\partial^{2} W}{\partial \theta^{2}}+\frac{\partial^{2} W}{\partial z^{2}}=0\end{array}\right.$

- Extrémité libre: $\left\{\begin{array}{c}\frac{\partial^{2} F}{\partial z^{2}}=0 \\ \frac{1}{R} \frac{\partial^{2} F}{\partial z^{2}}=0 \\ \frac{1}{R^{2}} \frac{\partial^{2} W}{\partial \theta^{2}}+v \frac{\partial^{2} W}{\partial z^{2}}=0 \\ \frac{1}{R^{3}} \frac{\partial^{3} W}{\partial \theta^{3}}+(2-v) \frac{1}{R} \frac{\partial^{3} W}{\partial z^{2} \partial \theta}=0\end{array}\right.$

Il est important de noter que lorsque le rayon tend vers l'infini mathématique, les équations (1) à (6) sont similaires à celles d'une plaque. C'est une preuve de la validité de notre modélisation.

\subsection{Méthodes mathématiques et expérimentale}

Les méthodes mathématiques utilisées visent à déterminer les fréquences propres de vibration des coques ainsi que la fréquence propre de la cavité du tambour.

Pour la détermination des fréquences propres de vibration des coques (sous-système 1), il est question de résoudre le système d'équations aux dérivées partielles (1) en tenant compte des conditions aux limites (4) à (6). Pour cela, on utilise la méthode de décomposition modale dans laquelle la déflexion de la coque est définie comme une combinaison linéaire des différents modes de vibration de fréquences différentes (chaque mode étant le produit d'une fonction dépendant du temps et d'une fonction dépendant de l'espace ; c'est-à-dire de l'angle $\theta$ et de la cote z). Il faut signaler que les termes non linéaires sont négligés. Pour plus de détails, voir les références (Donnell (1934), Von Karman and Tsien (1941), Talla Mbé (2007)).

Pour ce qui est de la détermination de la fréquence de vibration de la cavité du tambour (soussystème 2), elle est considérée comme un résonateur acoustique obéissant aux hypothèses de Helmhotz (Talla Mbé (2007)).
Pour ce qui est de la méthode expérimentale, un sonomètre et un fréquencemètre numérique ont été utilisés. Le sonomètre placé au niveau de la fente est connecté au fréquencemètre. Nous avons utilisé trois TAAs, fabriqués à partir du même bois, mais ayant des longueurs et des rayons différents. Pour chacun des TAAs, les sons ont été produits à plusieurs reprises, puis enregistrés par le sonomètre. La connexion entre le sonomètre et le fréquencemètre permet de lire chaque fois la fréquence du son produit. Les valeurs moyennes des fréquences données par le fréquencemètre ont été comparées aux résultats théoriques.

\section{Fréquences propres et formules} scientifiques du tambour d'appel

\subsection{Fréquences propres}

La résolution des équations (1) à (6) à partir de l'approche modale (Talla Mbé (2007)) permet d'établir que la fréquence propre d'une lèvre est donnée par la relation suivante :

$$
f_{\lambda, k}=\frac{h}{2 \pi L^{2}} \sqrt{\frac{E}{12 \rho\left(1-v^{2}\right)}} k^{2},
$$

où $\rho$ est la masse volumique du matériau constituant la lèvre. $k$ est un paramètre satisfaisant l'équation suivante:

$r_{1}\left[r_{2}^{2}+\lambda^{2} v\right]^{2} \tan \left(r_{2} \alpha\right)=r_{2}\left[r_{1}^{2}-\lambda^{2} v\right]^{2} \tanh \left(r_{1} \alpha\right)$

avec

$r_{1,2}=\sqrt{k^{2} \pm \lambda^{2}}, \alpha=\frac{R \beta}{L}$.

$\lambda$ caractérise la fréquence spatiale de vibration de la lèvre. Son expression est $\lambda=n \pi$ où nest un entier naturel ( $n=1,2,3, \ldots$ ). $\beta$ désigne l'angle d'ouverture de la lèvre.

De l'équation (7), on peut noter que la fréquence est proportionnelle à l'épaisseur de la lèvre et inversement proportionnelle au carré de sa longueur. Cette proportionnalité par rapport à l'épaisseur confirme les observations mentionnées en introduction : la lèvre de petite épaisseur émet un son grave caractéristique des faibles fréquences alors que la lèvre la plus épaisse produit un son aigu qui correspond aux fréquences plus élevées. 
Pour ce qui est du second sous-système qu'est la cavité considéré comme un résonateur acoustique, l'utilisation des hypothèses d'Helmhotz permet d'établir que l'expression de sa fréquence propre est :

$$
f_{0}=\frac{c}{2 \pi} \sqrt{\frac{e L}{h V}} \quad \text { (10) }
$$

où v esi le vuiume ue la cavile el c ld vilesse du son dans l'air.

Nous utiliserons ces expressions des fréquences caractéristiques pour établir les formules scientifiques qui, bien utilisées, optimisent la réponse acoustique du TAA et la gestion des intervalles musicaux.

\subsection{Formules scientifiques du TAA}

D ans un premier temps, nous admettons l'hypothèse selon laquelle le son caractéristique émis par le TAA est le fruit de l'interaction entre la lèvre vibrante et la cavité.

En effet, il faut que ces deux entités soient en résonance afin que la puissance sonore soit maximale. Cette hypothèse se traduit par $f_{0}=f_{\lambda, k}$. On en déduit que l'épaisseur de la lèvre est donnée par l'expression suivante :

$$
h^{3}=\frac{12 c^{2} \rho\left(1-v^{2}\right) L^{5}}{V E k^{4}} e
$$

L'équation (11) est la première formule scientifique du TAA. Elle permet de choisir une épaisseur appropriée en fonction de l'ouverture des fentes et vice-versa.

D ans un second temps, nous considérons que les lèvres femelle et mâle ont respectivement pour épaisseur $h_{1}$ et $h_{2}$. Cela permet de calculer ce qu'on appelle en musique intervallemisical qui est le rapport entre deux fréquences. De l'expression de la fréquence (voir équation (7)), on obtient que l'intervalle musical est donné par la relation suivante:

$$
K=\frac{f_{1}}{f_{2}}=\frac{h_{1}}{h_{2}}
$$

C'est la deuxieme iormue scienninque du TAA. Elle permet de produire des TAAs ayant des intervallesmisicaux différents. L'intervallemisical du TAA est généralement la tierce $\left(h_{1} / h_{2}=5 / 4\right)$.
Les formules (11) et (12) sont celles que doivent utiliser les artisans pour produire scientifiquement des TAAs ayant la puissance maximale et décider de la valeur de l'intervalle misical.

\subsection{Validation expénimentale}

Pour compléter le travail théorique, nous avons procédé à une validation expérimentale en faisant des mesures sur des prototypes de TAAs que nous avons fait fabriquer par un artisan du village E kalli sur la route Yaoundé-Mbalmayo au Cameroun (Figure 3).

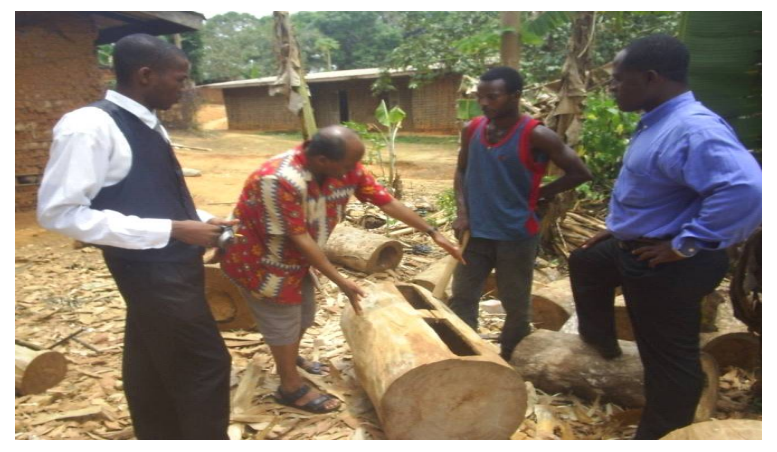

Figure 3: Quelques membres de l'équipe (projet Modernisation et miniaturisation du Tambour d'appel africain) échangeant avec un artisan (en démembré) du village Ekalli (route YaoundéMbalmayo, région du Centre, Cameroun) pour la commande des tambours respectant les formules scientifiques (de gauche à droite: D r. J.H. Talla Mbé en jacket noir, D r. F. Bingono Bingono tendant les mains sur le tambour et Prof. Paul Woafo en chemise bleue).

Pour mener à bien la phase expérimentale, nous disposons d'un sonomètre et d'un fréquencemètre numérique. Le sonomètre placé au niveau de la fente est connecté au fréquencemètre. Nous choisissons trois TAAs, fabriqués à partir du même bois, mais ayant des longueurs et des rayons différents. Les résultats sont consignés dans le Tableau 1. Les fréquences obtenues expérimentalement sont des moyennes des valeurs données par le fréquencemètre après production de plusieurs sons par le même TAA.

Les autres caractéristiques utilisées sont les suivantes : $\mathrm{E}=12 \mathrm{GPa}$ et $\nu=0,29, \rho=680 \mathrm{~kg} / \mathrm{m}^{3}$ et la vitesse $\mathrm{c}=330 \mathrm{~m} / \mathrm{s}$.

A partir de ce tableau, on peut constater, de manière globale, que l'écart entre les valeurs 
théoriques et expérimentales est inférieur à $15 \%$. Dans certains cas, l'erreur est inférieure à $5 \%$. On peut affirmer que l'approche théorique utilisée est acceptable. Une vérification de l'intenallemsical à partir de la formule (13) et des épaisseurs des lèvres a aussi permis de renforcer la validité de notre approche théorique.

Tableau 1: Tableau comparatif entre les valeurs numériques des fréquences obtenues à partir de l'approche théorique et celles obtenues à partir des mesures.

\begin{tabular}{|l|l|l|l|l|}
\hline $\begin{array}{l}\text { T ambour d'appel } \\
\text { africain (T AA) }\end{array}$ & Lèvre & $\begin{array}{l}\text { Fréquence obtenue } \\
\text { par la théorie (en } \\
H z \text { ) }\end{array}$ & $\begin{array}{l}\text { Fréquence obtenue par } \\
\text { l'expérience (en } \mathrm{Hz} \text { ) }\end{array}$ & $\begin{array}{l}\text { Ecart } \\
\text { (en \%) }\end{array}$ \\
\hline $\begin{array}{l}\text { Premier TAA } \\
\mathrm{L}=300 \mathrm{~mm}\end{array}$ & $\begin{array}{l}\text { M oins épaisse } \\
\mathrm{h}=6 \mathrm{~mm}\end{array}$ & 1173.76 & 1111.11 & 5.33 \\
\cline { 2 - 5 } & $\begin{array}{l}\text { Plus épaisse } \\
\mathrm{h}=7,5 \mathrm{~mm}\end{array}$ & 1467.20 & 1000 & 31 \\
\hline $\begin{array}{l}\text { D euxièmeT AA } \\
\mathrm{L}=400 \mathrm{~mm}\end{array}$ & $\begin{array}{l}\text { M oins épaisse } \\
\mathrm{h}=7 ; 5 \mathrm{~mm}\end{array}$ & 493.37 & 545.54 & 7.87 \\
\cline { 2 - 5 } & $\begin{array}{l}\text { Plus épaisse } \\
\mathrm{h}=9,375 \mathrm{~mm}\end{array}$ & 616.71 & 625 & 1.34 \\
\hline $\begin{array}{l}\text { T roisième TAA } \\
\mathrm{L}=500 \mathrm{~mm}\end{array}$ & $\begin{array}{l}\text { M oins épaisse } \\
\mathrm{h}=10 \mathrm{~mm}\end{array}$ & 484.95 & 416.66 & 14.08 \\
\cline { 2 - 5 } & $\begin{array}{l}\text { Plus épaisse } \\
\mathrm{h}=12,5 \mathrm{~mm}\end{array}$ & 581.94 & 500 & 14.08 \\
\hline
\end{tabular}

\section{Conclusion}

In fine, nous avons modélisé le tambour d'appel africain en convoquant la théorie des coques et des relations de Helmholtz. Les résultats théoriques ont été confirmés par des mesures expérimentales. D es formules scientifiques propres au tambour d'appel africain ont été établies. Celles-ci peuvent servir comme normes de fabrication afin d'obtenir des puissances sonores maximales et de gérer convenablement les intervalles misicaux. Des tests concluants ont été effectués sur des prototypes fabriqués en utilisant les formules obtenues.

Pour être faciles à utiliser par les fabricants des tambours, les formues données par les équations (11) et (12) devront être transformées en tables pour chaque espèce de bois approprié pour la fabrication des tambours. On établira alors des formules simples après avoir remplacé dans l'équation après avoir remplacé dans l'équation (12) les caractéristiques mécaniques et physiques que sont le module de Young $\mathrm{E}$, le coefficient de Poisson, la masse volumique, la vitesse $\mathrm{c}$ du son et le nombre $\mathrm{k}$ par leurs valeurs numériques.
Nous espérons que ces résultats préliminaires renforceront les travaux scientifiques sur cet instrument de musique et de communication. L'objectif final est de le moderniser et de le miniaturiser tout en fixant ses normes de fabrication. De plus, la modernisation pourra inclure des versions électroacoustiques et celles réalisées à partir des microcontrôleurs et des programmes informatiques. Plus loin, la miniaturisation pourra conduire à concevoir et fabriquer des tambours d'appel africain à plusieurs tons ou des tambours-balafons. Plus encore, cette contribution renforce les travaux scientifiques actuels portant sur le développement des instruments de musique virtuels (Bilbao et al. (2015), Leonard and Cadoz (2015), Vogl et al. (2017)).

\section{Références}

Alexandre, P. (1967). Langues et langage en Afrique Noire (Payot, Paris).

Bezukhov, N., Luzhin, O., Kolkunov N. (1987). Stability and dynamics of structures learnt by example. Mir Publishers Moscow. 
Bilbao, S, Torin, A. and Chatziioannou, V. (2015). Numerical modeling of collisisons in musical instruments. Acta Acustica united with Acustica 101, 155-173.

Donnell, E.H. (1934). A New theory for the buckling of thin cylindrical shells under axial compression and bending. Trans, ASME 56, 765806.

Ella, R. O. (2015). Description linguistique du shiwa, langue bantu du Gabon: phonologie, morphologie, syntaxe, lexique. Thèse de D octorat, Université Sorbonne Nouvelle Paris 3 et Museum National d'Histoire Naturelle.

Eno Belinga, M. S. (1966). Littérature et musique populaire en Afrique noire. Paris Ed. Cujas.

FAO (2002). Inventaire et utilisation des moyens traditionnels de communication au Centre, Sud, Est et Extrême-Nord du Cameroun. Food and Agricuture Organization (Rome).

Fame Ndongo, F. et collaborateurs (2010). Projet de Modernisation et Miniaturisation du Tambourtéléphone (Yaoundé, Cameroun).

Leonard, J. and Cadoz, C. (2015). Physical modeling concepts for a collection of multisensory musical instruments. Proc. Int. Conf. NIME'15, pp. 150-155, (Baton Rouge).

Sanders, Jr I.J. (1963). Nonlinear theories for thin shells. Q. Appl.Mash 21, 21-36.
Talla Mbé, J.H. (2007). Etude scientifique du tambour. Mémoire de DEA, Université de Yaoundé 1, Cameroun.

Thomas, O., Touzé, C., Chagne, A. (2005). Nonlinear vibrations of free-edge thin spherical shells : modal interaction rules and 1:1:2 internal resonance. Int. J. Solids and Structures 42, 33393373.

Vogl, R., D orfer, M., Widmer, G. and Knees, P. (2017). D rum transcription via joint beat and drum modeling using convolutional reccurent neural networks. Proc. 18th ISMIR Conf., pp. 150-157 (Suzhou).

Von Kàrmàn, T. and Tsien, H.S. (1941). The buckling of thin cylindrical shells under axialcompression. J. Aeronaut. Sci. 8, 303-312.

\section{Webographie}

Bingono-Bingono, F. (2018). Langue et musique - un mariage africain. Levure litteraire.com, ISSN 2268-9915, (en date du 20/ 06/ 2018). 